\title{
Attenuated periodical oscillation characteristics in a nanoscale particle-laden laminar flow
}

\author{
JiafengGeng ${ }^{1}$, Junwang Tang ${ }^{2}$, Yechun Wang ${ }^{1}$, Zhenxiong Huang ${ }^{1}$, Dengwei Jing ${ }^{1, *}$, Liejin Guo ${ }^{1}$ \\ 1. State Key Laboratory of Multiphase Flow in Power Engineering \& International Research Center for \\ Renewable Energy, Xi'an Jiaotong University, Xi'an, Shaanxi 710049, China \\ 2. Department of Chemical Engineering, University College London, Torrington Place, London, WC1E 7JE, UK \\ * Corresponding author: Tel.:+86-29-82668769; Email: dwjing@mail.xjtu.edu.cn
}

\begin{abstract}
Transport properties in nanoparticles-laden flow are of great importance for their practical use, such as in tubular reactor-based photocatalytic technologies. In our study, an experimental setup based on photocatalytic application was employed to investigate the nanoparticle transport characteristics in the flow reactor. An interesting periodical attenuated oscillation of the particle volume fraction and mean particle size with time was found in the nanoparticle-laden flow. We further analyzed the period of the attenuated oscillation curve by conducting a linear fitting, which shows that the period can be express as $T=f(Q, L)$ in which the period is proportional to the length of test section and inversely proportional to the flowrate. We found the time $5 \mathrm{~T}$ is a very important criterion for whether the particle suspension has reached the quasi-steady state and the nanoparticle has been well suspended. Our work is believed to be valuable efficient and effective transportation of nanoparticle in laminar flow with low energy consumption.
\end{abstract}

\section{Introduction}

The suspensions of micro- or nanoscale particles in liquid exist widely in various fields e.g. advanced material processing, electronic technology, chemical engineering, petroleum, food processing, waste treatment etc ${ }^{1-3}$. It is also the important media for some emerging technologies such as the enhanced heat transfer over nanofluid and photocatalytic energy conversion and environmental remediation over various nanoscale photocatalyst suspensions ${ }^{4-6}$. In all of these processes, particle transportation and deposition have significant impacts on the properties and 
thus functions of such kind of suspension. For example, in photocatalytic process, sedimentation of the photocatalyst leading to ineffective light absorption must be avoided ${ }^{7}$. In past researches, turbulence flow in the phtotocatalytic reactor is believed to be a necessary condition for good suspension of photocatalyst particles ${ }^{8}$. However, turbulence flow tends to consume much more energy than the laminar flow, which is a disadvantage for the overall energy efficiency of the solar photocatalytic water splitting process. Therefore, some researchers have tried to adopt laminar circulation flow in photocatalytic reactor ${ }^{9-11}$, which also shows good photocatalytic performance. Li Puma et al had indicated that the correspondence between the radiation field and fluid residence time is very important to the photocatalytic reaction ${ }^{11}$, which means the relatively low flowrate may be more benefit to the efficiency of photocatalytic reaction. Hence, it may suggest that the circulating particle-laden laminar flow is a suitable flow pattern of slurry photocatalytic reactor. However, few studies have revealed the transport phenomenon of polydisperse nanoscale particle-laden circulating laminar flow.

For the clearer understanding of the transport phenomenon in nanoparticle flow, many studies have been conducted by means of experimental. Sampling method as the most intuitive method has been employed to ascertain the axial and radial profiles of the solid concentration ${ }^{12}$. It is simple and reliable but also has the disadvantage of great dependence on sampling probe shape and location, and it could also bring some disturbances to the whole flowing suspension. Probe techniques have been widely used to study the particles concentration and movement in the solid-liquid suspension, including, for instance, impedance ${ }^{13}$, optical ${ }^{14}$, acoustic techniques ${ }^{15}$. All of these techniques are based on the different responses of electric, optical or acoustic signals between continuous and discrete phases which inevitably disturb the flow field due to the existence of probes. To overcome this defect, some non-intrusive methods have been developed, such as the light technique ${ }^{16}$, radiation technique ${ }^{17}$, ultrasonic attenuation techniques ${ }^{18}$, tomographic techniques ${ }^{19-21}$ etc. In the past decade, laser diffraction (LD) technique based on the particles diffraction has become a popular method to measure the particle size and concentration distribution $^{22,23}$, due to its high speed, good reliability and high reproducibility ${ }^{24}$. In general, the LD measurement is conducted after the suspension is in stable state, that is, the concentration and particle size distribution of the suspension hold an almost constant value. 
It is worth noting that in many photocatalytic reactors, nanoparticle suspensions are in fact circulated in a loop and such flowing reactors are cost-effective, easy for scale-up and for nanoparticles recycling6, 25-27. Obviously, the transport characteristics are significant to the performance of such kind of suspension reactor. The transport characteristics can be reflected in variation with time of some macroscopic parameters, such as concentration and particle size distribution. Segré $\mathrm{G}$ and Silberberg ${ }^{28}$ studied particles transport phenomenon in Poiseuille flow through a tube by blocking out of two mutually perpendicular light beams and found that the flow in tube tend to carry particles to a certain equilibrium position, irrespective of the radial position at which the sphere first entered the tube. Tirumkudulu et $\mathrm{al}^{29}$ reported a particle segregation phenomenon in a partially filled horizontal Couette device in which the suspension separates itself into alternating regions of high and low particle concentration along the length of the tube by employing a photographic method. Koh et $\mathrm{al}^{30}$ measured concentrated suspension flows by adopting Laser-Doppler anemometry technique and found that velocity profile was blunted while the concentration profile had a maximum near the center. Hampton et $\mathrm{al}^{31}$ studied demixing of neutrally buoyant suspensions of spheres under pressure driven flows in circular conduits. In our previous work, the photocatalyst itself was employed as a tracer particle for Particle Image Velocity (PIV) measurement combined with a new laser sheet image analysis (LSIA) technology to investigate both the particle velocity and number distribution in the tubular reactor ${ }^{32}$. In this study, an asymmetric velocity distribution of $\mathrm{TiO}_{2}$-water slurry flow was observed and a non-uniform particle distribution in the radial was found.

As mentioned above, photocatalytic water splitting hydrogen production is a very attractive technology for which one of the key issues is the well suspension property of the particles in the reactor $^{33-35}$. And the circulating flowrate is a very important effect which determines the suspending state of the slurry and then the photocatalytic reaction efficency ${ }^{36}$. In this study, an experimental setup based on photocatalytic application was employed to investigate the nanoparticle transport characteristics in the flow reactor. An interesting periodical attenuated oscillation of the particle volume fraction and mean particle size with time was found in the nanoparticle-laden flow. It was found that the periods of attenuated curve is proportional to the length of testing section and inverse proportional to the flowrate. However, a time of 5 periods is 
enough for the particles to reach the quasi-steady distribution in the laminar flow.

\section{Materials and experimental setup}

Figure 1a shows the experimental set-up designed in this study. It has eight parts: experimental section, observation window, inlet, outlet, centrifugal pump, wet sample injector, laser particle analyzer, connecting tube. The experimental section in this study is made of acrylic glass with an inner diameter of $30 \mathrm{~mm}$ and outer diameter of $40 \mathrm{~mm}$. Because the length of experimental section is an important factor, a multi-stage detachable structure that can be adjusted according to the required length, has been designed to investigate the effect of the tube length on the flow characteristics. The detachable tubes are all $0.4 \mathrm{~m}$ long. They are transparent and can be connected by threaded looper sealed with flat gasket. An inlet and outlet were also designed to ensure that the experimental section forms a loop with the rest of the components, both of which are $0.1 \mathrm{~m}$ long. It has been proposed that assembly difficulties and strength problems could occur if the glass tube is more than 2 meters in length for outdoor use ${ }^{6}$. Therefore, the maximum tube length in this study is set as $2.2 \mathrm{~m}$, including a $2 \mathrm{~m}$ long experimental section consisting of 5 detachable tubes and the inlet and outlet sections mentioned above. And if only 1,2,3 and 4 detachable tubes are used, the lengths of experiment section are $0.6 \mathrm{~m}, 1.0 \mathrm{~m}, 1.4 \mathrm{~m}$, and $1.8 \mathrm{~m}$, respectively. In fact, these lengths of the experiment section have also been employed in other works ${ }^{25,27}$. A cuboid observation shell was designed and installed on the experimental section to observe the particle state. In order to avoid aberration caused by the cylindrical tube, an acrylic jacket with cuboid shape was designed, which is wrapped outside the pipe and filled with sodium iodide solution to compensate for the refractive index error caused by acrylic material ${ }^{37}$. Two removable quartz glass windows are also assembled on the cuboid shell to make sure a clear observation. The centrifugal pump is used to control the flowrate accurately to $10 \mathrm{~L} / \mathrm{h}, 15 \mathrm{~L} / \mathrm{h}, 20 \mathrm{~L} / \mathrm{h}, 25 \mathrm{~L} / \mathrm{h}$ and $30 \mathrm{~L} / \mathrm{h}$, respectively. And the Reynolds number corresponding to the 5 flowrates are 58.95, 88.42, 117.89, 147.36 and 176.84, respectively, and all of them are less than 2020 which is considered as the lower critical point of the transition to turbulence in pipe flow ${ }^{38}$. A wet sample injector was set to add the particles to the circulating flow system and also as a reservoir with mixer. A Malvern Spraytec 2000 laser particles analyzer was used to measure particle concentration and particle size distribution of the added particles in the size range of $0.1-2000 \mu \mathrm{m}$ simultaneously. In addition, as the whole system 
needs to be closed into a loop, some connecting tubes are used to connect the above components. And the inner diameter of the connecting tubes which are made of silicone is $8 \mathrm{~mm}$, and the total length of them is $2.3 \mathrm{~m}$. It is worth noting that there is a diameter difference between the connecting tube and the main tube and this difference is supposed to have impact on the flow flied. However, considering that in practical application, it is a common situation to connect components with different tube diameters. It is thus considered that our experimental system that has an enough total tube length can still reasonably exhibit the common phenomena existing in a particle-laden circulating flow.

Figure $1 \mathrm{~b}$ shows the complete experimental system with the longest experiment section length of $2.2 \mathrm{~m}$. Degussa P25 titanium dioxide was employed as model particles and main parameters for this widely used photocatalyst can be found in literature ${ }^{39}$. According to the literature, $\mathrm{P} 25 \mathrm{TiO}_{2}$ powder has an primary particle size of $21 \mathrm{~nm}$ and a true density of $3900-4000 \mathrm{~kg} / \mathrm{m}^{3}$. In our design, the apparent volume fraction of nanoparticles is $\varphi=1 \times 10^{-4}$ in the whole system, corresponding to the mass concentration of $0.4 \mathrm{~g} / \mathrm{L}$ according to the density of $\mathrm{P} 25 \mathrm{TiO}_{2}$. This value of concentration is generally employed in the photocatalytic reaction ${ }^{40}$ and thus has been considered in our study.

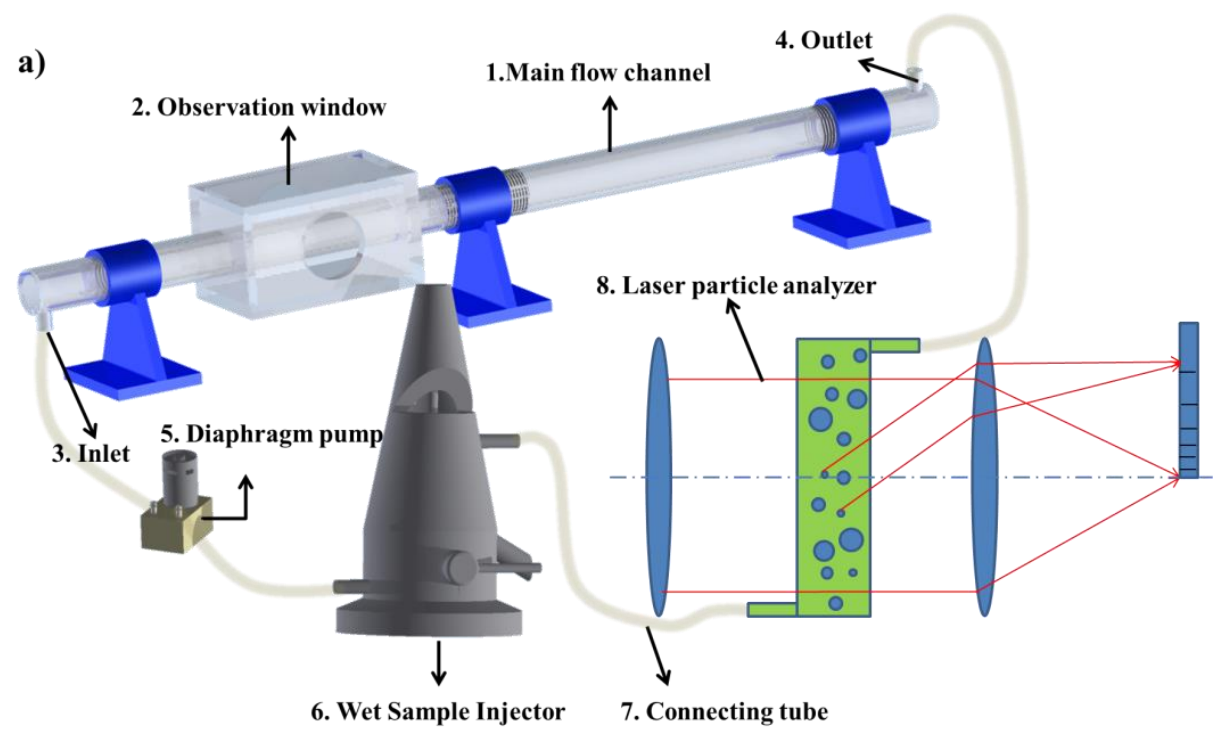




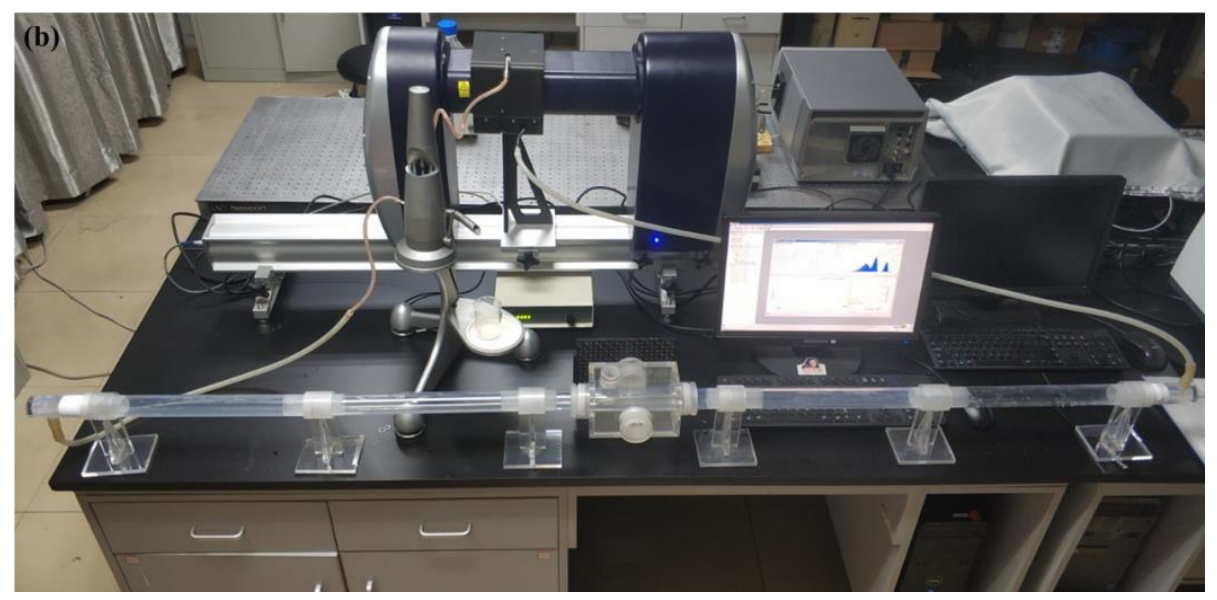

Figure 1. (a).Schematic of experiment set-up (b). Photo of the experiment set-up (tube length is 2.2 m)

\section{Results and discussion}

Dynamic particle size distribution characteristic in the flowing suspension. $0.4 \mathrm{~g} / \mathrm{L}$ P25-water suspension ( $(=0.01 \%)$ was prepared firstly for the following study. The particle size distribution for P25 in water was measured by laser diffraction (LD) method. As shown in Figure 2a, the particle size distribution (PSD) is in a wide range $0.1-100 \mu \mathrm{m}$ and there are three peaks in the particle distribution. Obviously, the nanoscale primary particle with original size of $21 \mathrm{~nm}$ has aggregated into large secondary particle of various sizes. It is worth mentioning that aggregation is a very common phenomenon in nanoscale particle suspension, which is related to many factors, such as the temperature of suspension, the $\mathrm{pH}$ of the suspension, particle size, shape, method of preparation and solid concentration ${ }^{4}$. In this study, parameters that could affect the aggregation process have been controlled strictly in the below experiment, including the suspension composition, solid concentration, temperature, method of preparation. In addition, the suspensions used in different conditions in our study were prepared in one batch, so that the initial particle size distribution is the same for all cases. The left hand side small peak corresponds to the particle diameter of below $1 \mu \mathrm{m}$, the middle one is between $1-20 \mu \mathrm{m}$, and the right hand side one is between 20-100 $\mu \mathrm{m}$. Accordingly, the particles size distribution in the fluid can be roughly divided into three ranges: the small particles with a particle size range of $0.1-1 \mu \mathrm{m}$, the medium-sized particles with a range of $1 \mu \mathrm{m}-20 \mu \mathrm{m}$, and the large particles with a size range of $20 \mu \mathrm{m}-100 \mu \mathrm{m}$. In the following section, unless otherwise stated, the small, medium-sized and large particles 
correspond to above size ranges, respectively.

After the prepared P25 suspension has been added in the circulating system, the laser particle analyzer was started to measure the particles volume fraction and size distribution simultaneously. Figure $2 \mathrm{~b}$ shows the variation of mean particle size and particle volume fraction with time in the circulating flow system. Here, the two curves with the arrow pointing to the left coordinate axis are the variations of two kinds of mean particle sizes, $\mathrm{D}_{32}$ and $\mathrm{D}_{43}$ with time, respectively. And the curve with the arrow pointing to the right coordinate axis is the variation of particles volume fraction with time. Here, $\mathrm{D}_{32}$ is also called Sauter mean diameter and it is the mean particle size taking into account both volume and surface area of the particles ${ }^{41}$, while the $\mathrm{D}_{43}$ is the mean particle size based on the volume moment ${ }^{42}$. The expressions for the two average particle sizes are as follows:

$$
\begin{gathered}
\boldsymbol{D}_{32}=\frac{\sum n_{i} D_{i}^{3}}{\sum n_{i} D_{i}^{2}} \\
\boldsymbol{D}_{43}=\frac{\sum n_{i} D_{i}^{4}}{\sum n_{i} D_{i}^{3}}
\end{gathered}
$$

where the variable $n_{i}$ and $D_{i}$ are, respectively, the number frequency and diameter of particulate matter in a particulate size fraction. Both mean particle sizes and particle volume fraction exhibit an obvious periodical attenuated oscillation tendency with time as can be noted in Figure 2b. In particular, the curve shows significant oscillation amplitude in the first 5 periods, and after that the oscillation of the curve becomes almost invisible and it means that the volume fraction of the particles in suspension tends to be a constant value. It indicates that the nanoparticles transport in the flowing suspension and achieve uniform distribution gradually which can be considered as a quasi-steady state of the circulating particle-laden flow. Here, the quasi-steady state means that such macroscopic parameters for the slurry as the particle volume fraction and particle mean diameter tend to be constant while it is still in a flowing state. In general, $\mathrm{D}_{43}$ represents the existence of larger particles while $\mathrm{D}_{32}$ is associated with the smaller particles. A big difference between $\mathrm{D}_{32}$ and $\mathrm{D}_{43}$ often means that the particle size has a bimodal distribution, while a small different between $\mathrm{D}_{32}$ and $\mathrm{D}_{43}$ often means that the size distribution have only one peak ${ }^{43}$. From 
Figure $2 b$, one can find that the curve of $\mathrm{D}_{43}$ is always higher than that of $\mathrm{D}_{32}$, indicating that particle size distribution in the circulating flow may exhibit a bimodal distribution. Interestingly, there is an obvious difference between the two oscillation curves of the mean particle size and the concentration curve. The former two curves of mean particle size show an overall upward trend with the passage of time, while the volume fraction curve oscillates repeatedly near an almost constant value.
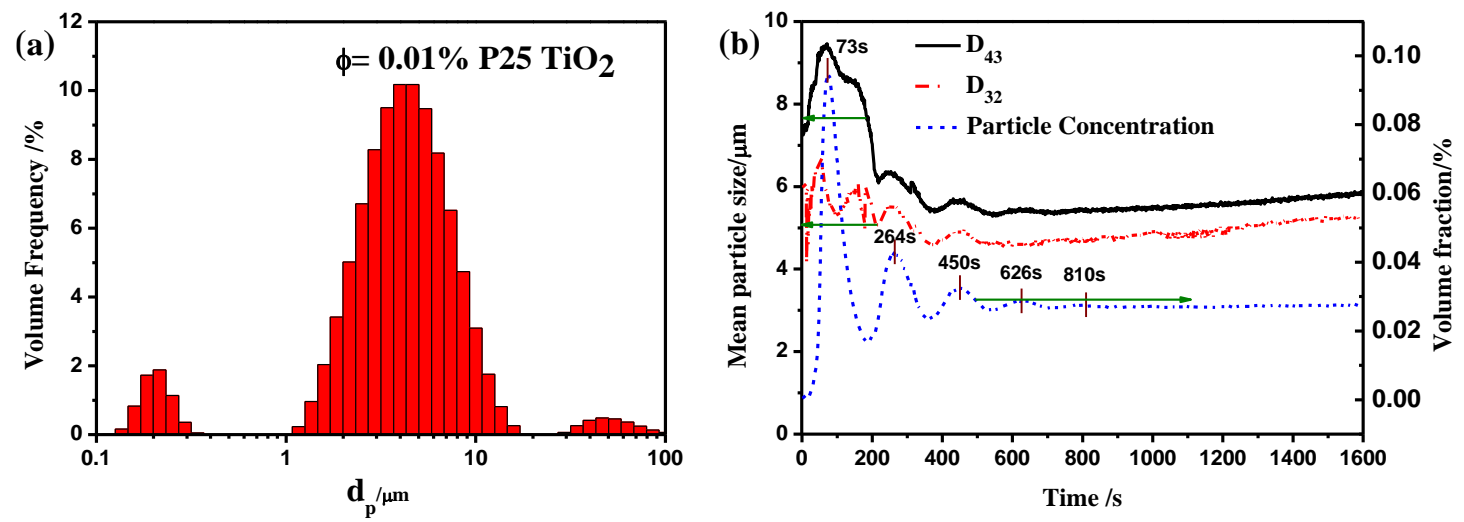

Figure 2. (a) Static particles size distribution of the $\mathrm{P} 25-\mathrm{TiO}_{2}$ powder dispersed in water, (b) Variation of various mean particle sizes and particle volume fraction with time in circulated flow.

Variation of particle size distribution with time in the flowing suspension. Figure 3 shows the variation with time of the volume particle size distribution and number particle size distribution in the circulating system with the experiment section length of $1.4 \mathrm{~m}$ and the flowrate of $20 \mathrm{~L} / \mathrm{h}$. Here, the times corresponds to the five peaks on the particle volume fraction curve in Figure $2 \mathrm{~b}$ and they $\operatorname{are} 73 \mathrm{~s}, 264$ s, 450 s, 626 s, 810 s, respectively. Time point of $1 \mathrm{~s}$ and $1000 \mathrm{~s}$ were also selected which were considered as the beginning state and quasi-steady state, respectively. It is also worth noting that the volume particle size distribution is based on the volume occupied by particles in suspension, while the number particle size distribution is based on the quantity of particles, and there is a relationship between the two kinds of size distribution, as follows:

$$
V(D)=\frac{1}{6} \pi D^{3} N(D)
$$

where $V(D)$ and $N(D)$ are, respectively, volume distribution and number distribution, and D is the 
diameter of particle. It can be seen that the particle volume is the cubic relationship of the diameter, thus the presence of a few large particles can have a significant impact the volume distribution.

From Figure $3 \mathrm{a}$, one can note that the volume particle size distribution exhibits unimodal distribution at the first two selected times, i.e., at $1 \mathrm{~s}$ and $73 \mathrm{~s}$, while it turns into bimodal distribution after $73 \mathrm{~s}$. And the medium-sized particles occupy the most volume frequency in the volume particle size distributions at all times, for the peaks with the range of 1-20 $\mu \mathrm{m}$ keep the highest in the volume particle size distribution at all times. Although the volume particle size distribution at $\mathrm{t}=1 \mathrm{~s}$ and $73 \mathrm{~s}$ are both unimodal, it is obvious that the particle size distribution is wider at $73 \mathrm{~s}$ than that at $1 \mathrm{~s}$. And the volume particle size distribution at $73 \mathrm{~s}$ covers all the range of particles. However, there are only medium-sized particles at $\mathrm{t}=1 \mathrm{~s}$. According to the design of our set-up, $\mathrm{t}=1 \mathrm{~s}$ is the time that first group of particles can be detected by the laser particle analyzer. It indicates that the medium-sized particles have better tracking characteristics. Particularly, tracking characteristics is very important for selecting tracer particles in PIV technology, in which the particle velocity lag should be smaller and the relaxation time should be shorter ${ }^{44}$. From the perspective of photocatalytic reaction, this characteristic is also very important, because it represents whether the particles can be effectively transported and therefore whether the particles settle in the reactor tube.

From inset 1 of Figure $3 \mathrm{a}$, one can find that the small particles occupy very little volume frequency in the circulating flow system at any time, while it reaches maximum value at $t=264 \mathrm{~s}$. From inset 2 of Figure 3a, one can see that the position of the peak representing the medium-sized particles shifts to left after $t=73 \mathrm{~s}$ and it changes relatively intense from $t=73 \mathrm{~s}$ to $t=450 \mathrm{~s}$ and less obviously after $\mathrm{t}=450 \mathrm{~s}$. Comparing the three volume particle size distribution at $\mathrm{t}=73 \mathrm{~s}, 264 \mathrm{~s}$ and $450 \mathrm{~s}$, it is not difficult to find that the particle size distribution at $\mathrm{t}=73$ is a unimodal distribution, while that of $\mathrm{t}=264 \mathrm{~s}$ and $450 \mathrm{~s}$ are bimodal distribution, but the particles at $\mathrm{t}=264 \mathrm{~s}$ are small particles and medium-sized particles, the particles at $\mathrm{t}=450 \mathrm{~s}$ are medium-sized particles and large particles. From inset 3 of Figure 3a, one can note that the peak of large particles doesn't appear until $\mathrm{t}=626 \mathrm{~s}$, and the height of the peak increase with time. As has been mentioned above, $\mathrm{t}=1000 \mathrm{~s}$ is considered as the quasi-steady state for the particle volume fraction in the circulating system becoming a constant value after this time. It can be seen that the volume particle size distribution at this time still shows a bimodal distribution, and the two peaks correspond to 
medium-sized and large particles respectively and the corresponding particle sizes are about $5.5 \mu \mathrm{m}$ and $60 \mu \mathrm{m}$ respectively.
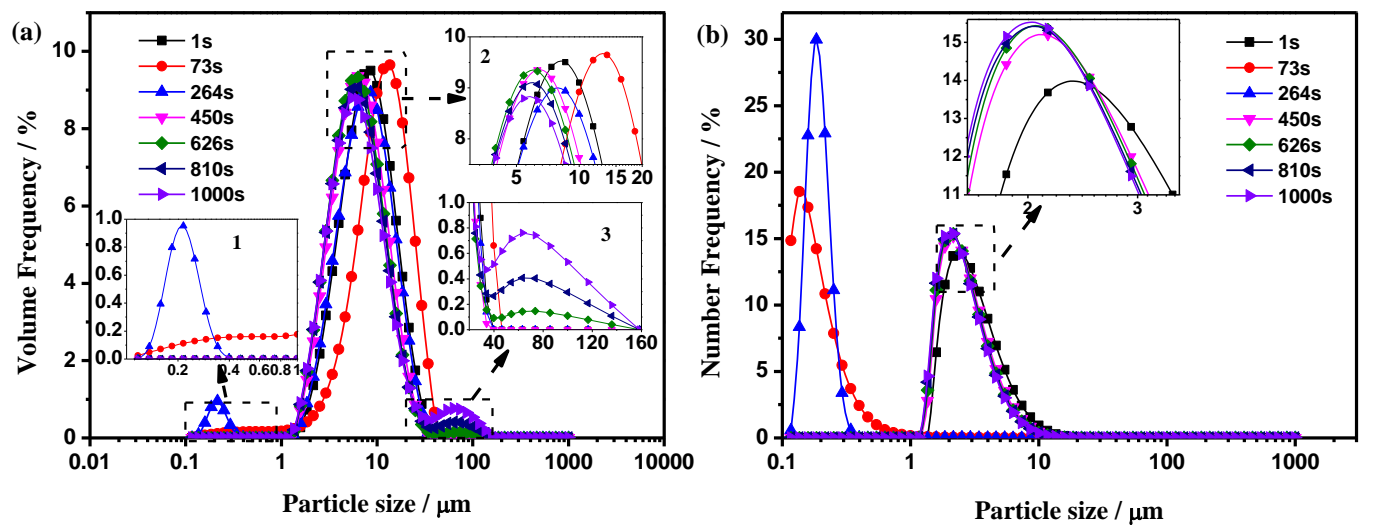

Figure 3. (a) Volume Frequency and (b) Number Frequency against particle size in the circulating system recorded at various time, the experiment section is $1.4 \mathrm{~m}$ long and the flowrate is $20 \mathrm{~L} / \mathrm{h}$.

Figure $3 \mathrm{~b}$ shows that the peak of the number particle size distribution at $\mathrm{t}=73 \mathrm{~s}$ and $264 \mathrm{~s}$ are about $100 \mathrm{~nm}$ and $250 \mathrm{~nm}$, respectively. In addition, it is obvious that the number particle size distributions at $\mathrm{t}=1 \mathrm{~s}, 450 \mathrm{~s}, 626 \mathrm{~s}, 810 \mathrm{~s}, 1000 \mathrm{~s}$ are all unimodal distribution and the peaks are all in range of 2-5 $\mu \mathrm{m}$. To understand this phenomenon, particle size distribution from the perspective of volume and quantity needs to be further discussed. For example, assuming that all the particles are spherical, if the particle size of a larger particle is 10 times that of a small particle, its volume will be 1000 times that of a small particle according to Equation 3. Therefore, it can be easy to understand why there is only a small peak for the small particles in the volume particle size distribution at $\mathrm{t}=73 \mathrm{~s}$ and $264 \mathrm{~s}$, while a very high peak for the small particles in the number particle size distribution at these two times, considering that the large amount of small particles only occupy a very small volume. On the other hand, large particles appears only after $\mathrm{t}=450 \mathrm{~s}$ and shows a small peak in the volume particle size distribution, but it almost has no proportion in the number particle size distribution. This phenomenon indicates that the large particles are in the minority in the suspension flow while it can influence the volume particle size distribution very significantly. Furthermore, large proportion of medium-sized particles both from the volume and quantity point proves that this part of particles prevail in the circulating flow. Also, this part of particles is preferred for the photocatalytic reaction for their larger surface area and better light absorption characteristics ${ }^{45}$. In particular, we assume that the medium-sized particles can be well 
suspended in the laminar flow. This may have certain significance to increase the overall energy conversion efficiency of the solar photocatalytic water splitting system, for the laminar flow consume less energy than turbulence flow.

Photo of the particle-laden laminar circulating flow in the tube. As mentioned above, there is an observation window in the experimental device to observe the movement of particles. Figure 4a shows the first photo that was taken when the first part of particles reached the observation window. Again, it can be seen that the particles group presents a parabolic profile, indicating their distribution in a laminar flow. In our previous work, the velocity field of this kind of suspension has been measured by PIV, which shows a parabolic distribution but the axis of symmetry is not in the center of the tube ${ }^{32}$. And it can be seen that the axis of symmetry in Figure 4a is lower than the center line as marked by the red dotted line. Figure $4 \mathrm{~b}$ shows the concentrated particles reaching the observation window which corresponds to the first peak in the particle volume fraction oscillation curve in Figure 2b. Figure 4c shows the time when the concentrated particles have just passed through the observation window which is assumed as corresponding to the first valley value at $\mathrm{t}=185 \mathrm{~s}$ of the particles volume fraction oscillation curve in Figure $2 \mathrm{~b}$. This assumption was based on the view of the observation window which appears a very light region compared with Figure 4b. Figure 4d shows the image time when the particles arranged in parabola reached the observation window again, which can be seen as the beginning of the second peak of particles volume fraction oscillation curve in Figure 2b. Figure 4e shows image when the concentrated particles corresponding to the second peak in the particles volume fraction oscillation curves in Figure 2b, reached the observation window. Visibly, the color in Figure 4e is lighter than Figure $4 \mathrm{~b}$, which means the particle volume fraction in Figure $4 \mathrm{e}$ is less than that in Figure $4 \mathrm{~b}$ according to Lambert-Beer law $^{46}$. This is consistent with the results that the particle volume fraction curve exhibits an attenuated oscillation in Figure 2a. Figure $4 \mathrm{f}$ shows the state when the particle volume fraction becomes uniform and reaches the quasi-steady state. From Figure 4a-f, it can be further concluded that the particle concentration and particle size oscillation phenomenon in circulating flow is closely related to the particles movement following the fluids which has a parabolic velocity distribution. 

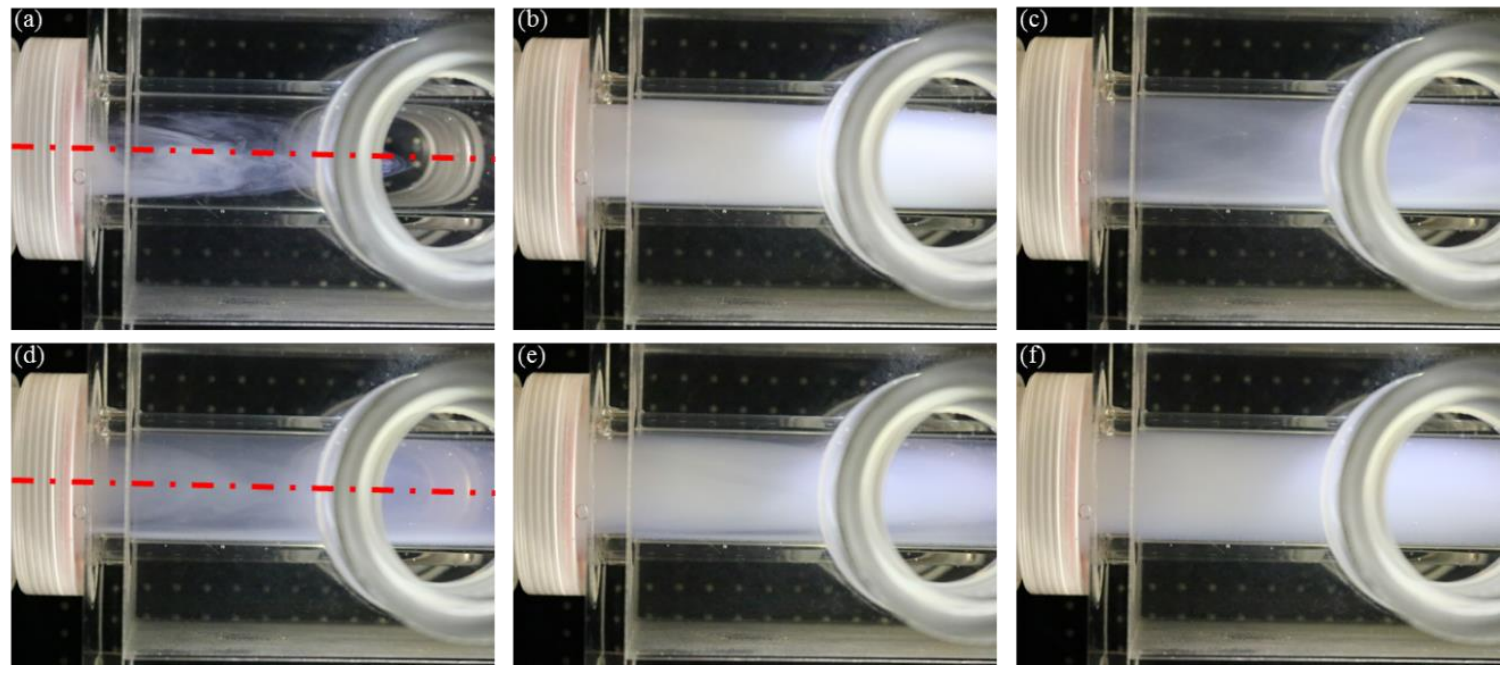

Figure 4. Photos of particles transport phenomenon in the circulating flow in the experiment section which the length is $0.6 \mathrm{~m}$ and the flowrate is $15 \mathrm{~L} / \mathrm{h}(\mathrm{a}) \mathrm{t}=1 \mathrm{~s},(\mathrm{~b}) \mathrm{t}=22 \mathrm{~s},(\mathrm{c}) \mathrm{t}=39 \mathrm{~s},(\mathrm{~d}) \mathrm{t}=50 \mathrm{~s}$, (e) $59 \mathrm{~s},(\mathrm{f}) 123 \mathrm{~s}$

\section{Influence of the flowrate of suspension on particle volume and particle size Figure}

5 shows the influence of flowrate on the particle volume fraction in circulating flow. It can be found that all the oscillation curve of particles volume fraction under different flowrates are very similar, the main difference is the time interval between peaks of oscillation. For the sake of the following discussions, we defined the time interval as the period of the oscillation curve. And it can be intuitively seen that the period tends to decrease with the flowrate increasing. In particular, the particle volume fraction at quasi-steady state is very different for different flowrates, and it can be seen that the particle volume fraction at $20 \mathrm{~L} / \mathrm{h}, 25 \mathrm{~L} / \mathrm{h}$ and $30 \mathrm{~L} / \mathrm{h}$ is close, while the particle volume fraction at $10 \mathrm{~L} / \mathrm{h}$ and $15 \mathrm{~L} / \mathrm{h}$ is much lower than that at the above three flowrates. 


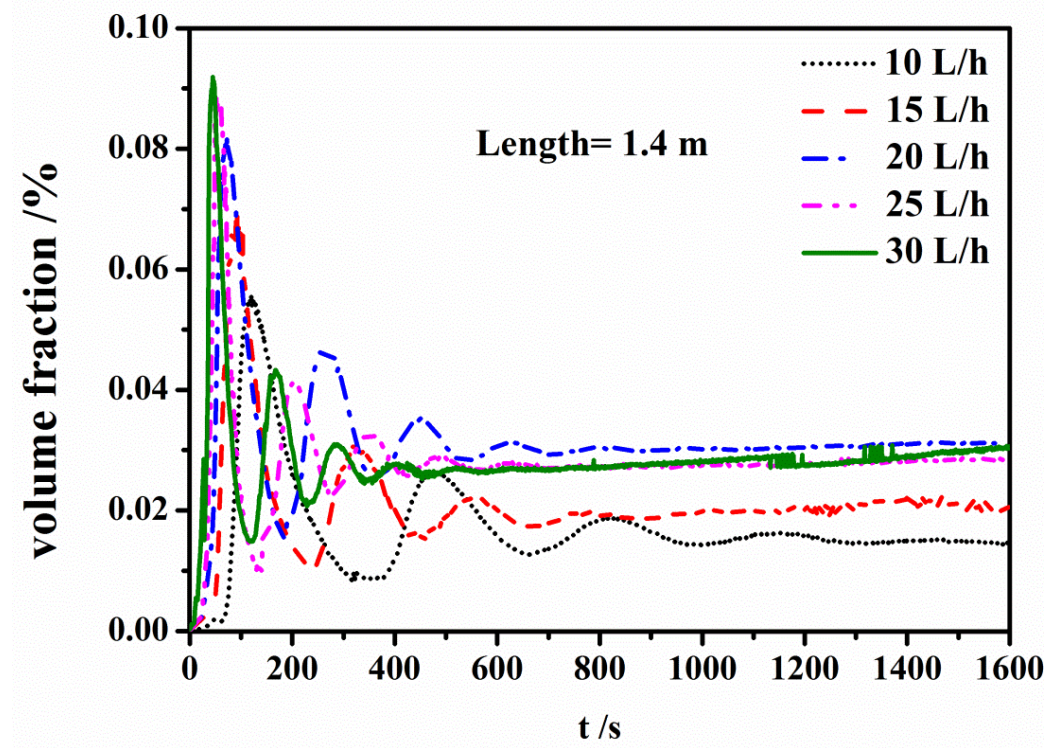

Figure 5. Influence of the flowrate on the particles volume fraction oscillation curve

The mean particle size and the volume particle size distribution at the quasi-steady state versus the various flowrates are shown in Figure 6. From the Figure 6a, it can be noted that the $\mathrm{D}_{43}$ firstly decreases from the flowrate $10 \mathrm{~L} / \mathrm{h}$ to $15 \mathrm{~L} / \mathrm{h}$ and then increases obviously with increasing flowrate, while $\mathrm{D}_{32}$ changes only a little. As has been discussed above, large particles contribute significantly to the value of $\mathrm{D}_{43}$. Accordingly, it indicates that the large particles are easier to be transported in the circulating system with high flowrates. It can be also found that the difference between $\mathrm{D}_{43}$ and $\mathrm{D}_{32}$ reach the maximum at flowrate $30 \mathrm{~L} / \mathrm{h}$, which can also prove that the particles distribution will be wider at larger flowrate. Figure $6 \mathrm{~b}$ shows that the equilibrium particles size distributions for all the five different flowrates exhibit a bimodal distribution. The peak corresponding to the large particles increases with the flowrate increasing. This phenomenon is in consistent with the result in Figure 6a, for the large particles are easier to settle than the other two kinds of particles. As mentioned above, particle volume fraction at lower flowrate such as 10 $\mathrm{L} / \mathrm{h}$ is obviously lower than that at higher flowrate, and it can be attributed to the sedimentation of larger particles. Sedimentation of larger particles means the loss of photocatalysts, so a relative higher flowrate is required to guarantee that most of the particles can be transported in the circulating system. Nevertheless, it can be found that the mean particle size at flowrate $10 \mathrm{~L} / \mathrm{h}$ is even larger than at $25 \mathrm{~L} / \mathrm{h}$. Compared with Figure $6 \mathrm{~b}$, this phenomenon can be attributed to the 
overall right-hand side movement of the volume particle size distribution at $10 \mathrm{~L} / \mathrm{h}$. It indicates that the medium-sized particles should occupy larger proportion in the volume particle size distribution compared to the other cases. And this phenomenon also exhibit that, according to our study, if the particle size can be controlled less than $20 \mu \mathrm{m}$, a very small flow rate such as $10 \mathrm{~L} / \mathrm{h}$ can ensure the effective suspending of particles.
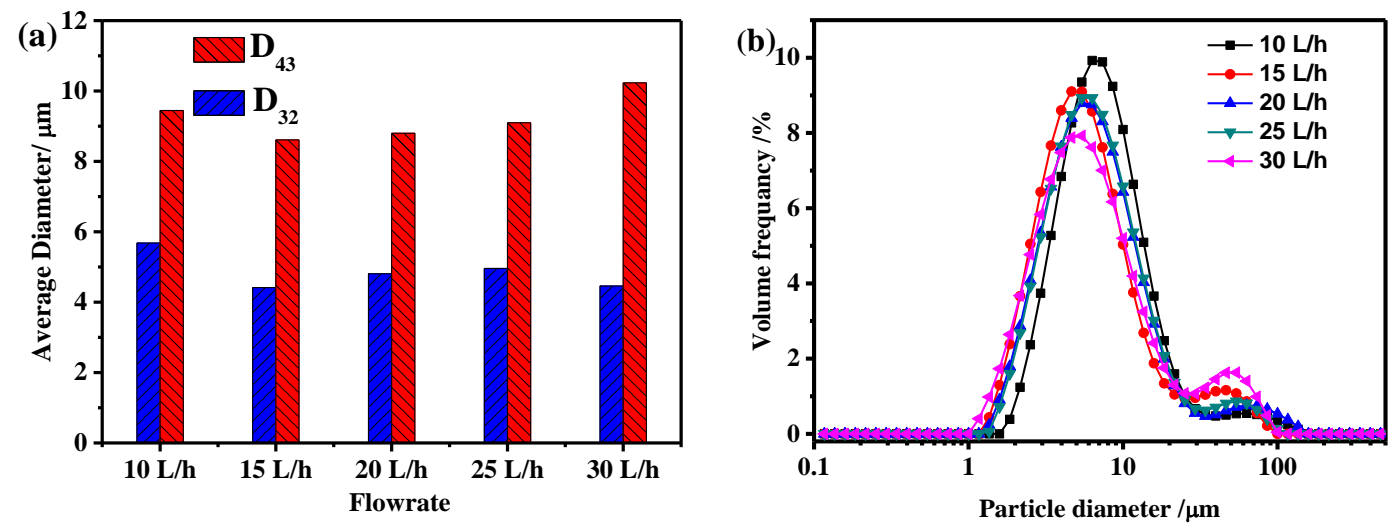

Figure 6. Variation of (a) mean particle size with flowrate, (b) volume distribution of particle size with flowrate, when particle concentration reaches quasi-steady state

\section{Influence of the tube length on particle volume fraction and particle size For the} tubular reactor, due to the requirement of light transmittance, the diameter of the reaction pipe is generally small, so a relative longer tube length is needed to ensure sufficient light area. On the other hand, tube length could increase the particle transport distance which will in turn influence the particle distribution characteristics, thus study the influence of tube length is important. Figure 7 shows variation of the particle volume fraction in the circulating system with different tube length at flowrate of $20 \mathrm{~L} / \mathrm{h}$. It can be found that all of the five particle volume fraction curves have the periodic attenuated oscillation phenomenon, but the periods of the five curves increase with the increasing of tube length. In addition, it can be observed that the particle volume fraction at quasi-steady decreases as the tube length increases. 


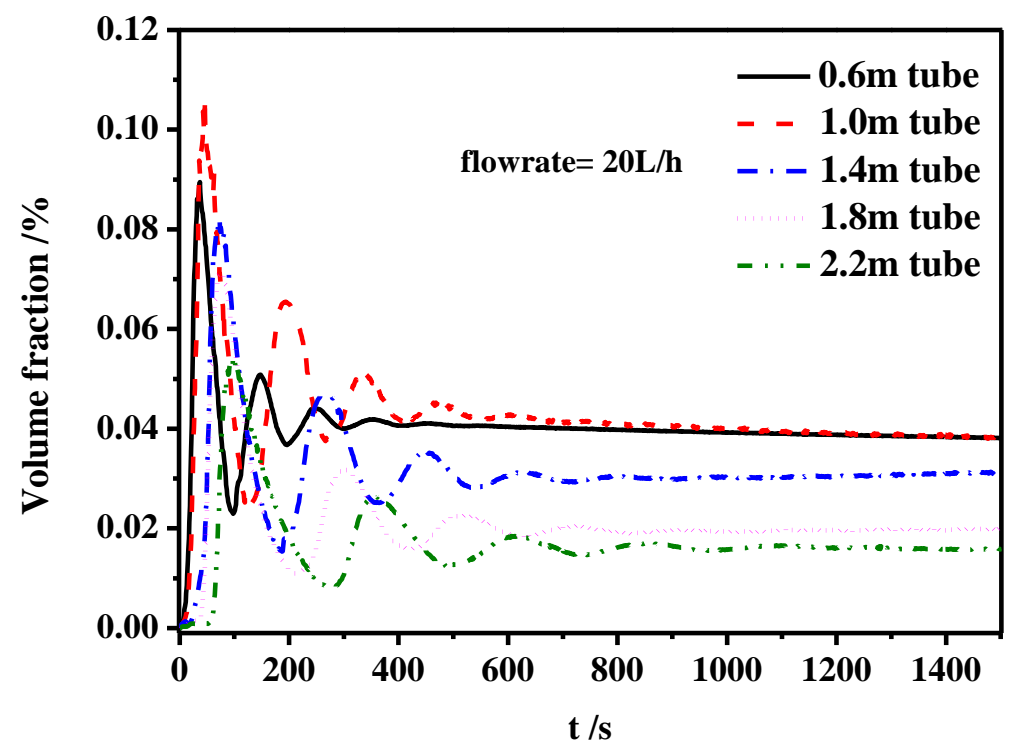

Figure 7. Influence of the experiment section length on the particles volume fraction oscillation curve

Influence of the experiment section length on the particle size in the particle transport process is shown in Figure 8. It can be found from Figure 8a that the mean particle size tends to decreases with the length of experiment section increasing, especially for the mean particle size $\mathrm{D}_{43}$. As has been mentioned above, $\mathrm{D}_{43}$ is very sensitive to the large particles, so it can be derived that the proportion of large particles decreases as the length of experiment section increases. Figure $8 \mathrm{~b}$, shows similar phenomenon. Not only the peak of large particles but also the peak of medium-sized particles moves to left hand side as the length of experiment section increases.
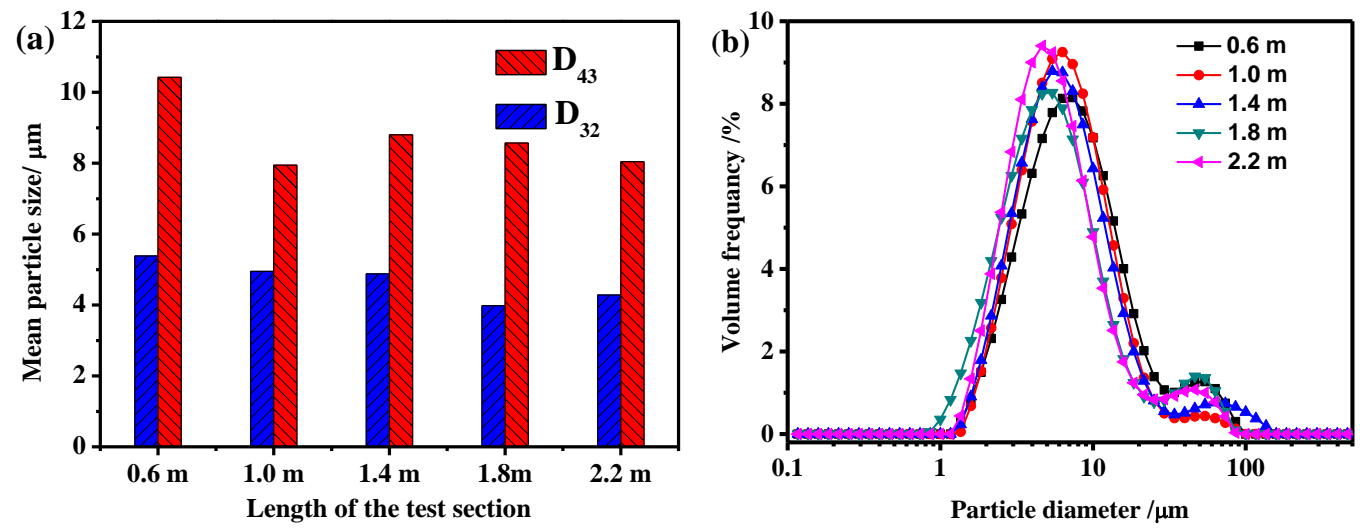

Figure 8. Influence of experiment section length on (a) variation of mean particle size, (b) variation of volume distribution of particle size, when the particles movement reaches the quasi-steady state 
Experimental data fitting and analysis From the Figures 2, 5, and 7, one can find that the attenuated oscillation exists at any flowrate and in the experiment section with any length. Also, Figure 4 further shows that the attenuated oscillation phenomenon is likely due to the parabolic profile flow field in laminar flow. With this kind of flow field, particles which are near the center line of tube tend to have higher velocity, while particles which are near the wall of tube tend to have lower velocity, and this phenomenon has also been used to measure the particle size and separate different supramolecular species that is called field-flow fractionation ${ }^{47}$. And it can be assumed from Figure 5 that the period of the attenuated oscillation might be inversely proportional to the flowrate, whereas it can be deduced from Figure 7 that the period of the attenuated oscillation might be proportional to the length of experiment section. To further confirm the above relations, two linear fit curves which show the relationship between the reciprocal of flowrate, the length of the experiment section and the period of the attenuated curve are given in Figure 9 a and $\mathrm{b}$, respectively. And the $\mathrm{R}^{2}$ values of the two curves are both larger than 0.99 , proving the good linear relationship.
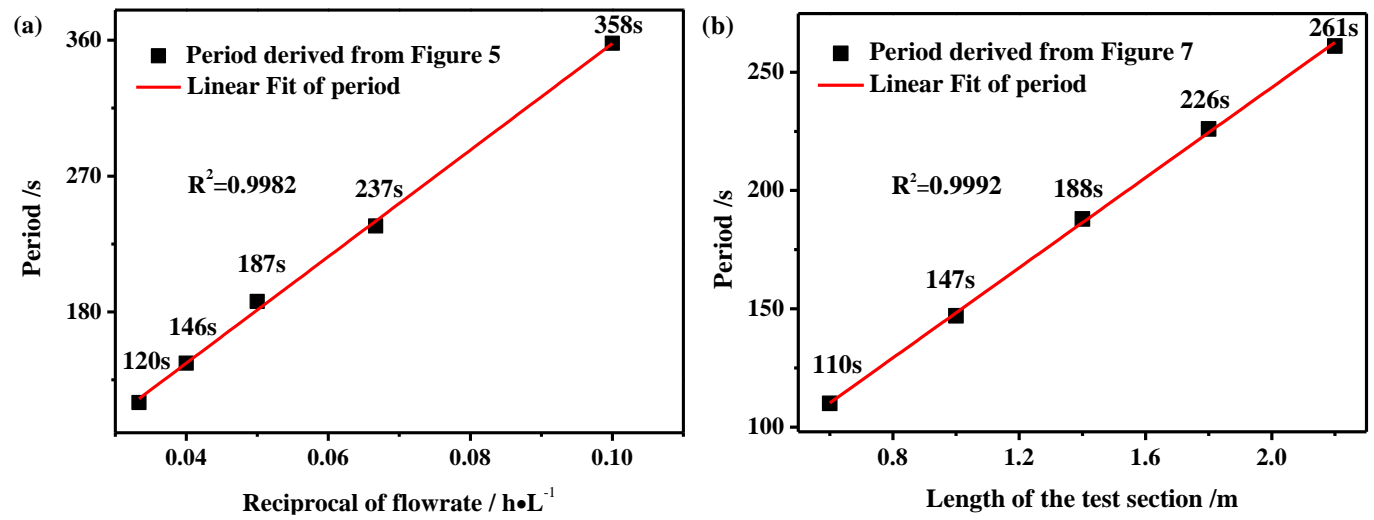

Figure 9. Linear fit of the relationship between (a) reciprocal of flowrate, (b) length of the experiment section and the period of the attenuated oscillation

Apart from the linear relationship of the periods, one can also find that the attenuated oscillation curve tend to be a flat line after five periods for all the conditions. This enlightens us that a time of five periods can be an important criterion for the quasi-steady state of the suspension flow. As mentioned above, if the period of the attenuated oscillation, the flowrate and the length of the experiment section are defined as T, Q and L, respectively, the relationship between them can be 
expressed as the following equations according to Figure $9 \mathrm{a}$ and $\mathrm{b}$ :

$$
\begin{aligned}
& T=\frac{3528.76}{Q}+4.93 \\
& T=95.25 L+53.05
\end{aligned}
$$

In the process of experiment, two factors, i.e. the length of the experiment section and the flow rate, are considered respectively, so it is natural to think whether the two linear relations can be unified into one equation, that is, written in the form of $T=f(Q, L)$. Noted that the flowrate in Equation 4 is $20 \mathrm{~L} / \mathrm{h}$, while the length in Equation 5 is $1.4 \mathrm{~m}$, consequently, the two equations can be combined in an approximate expression as follows:

$$
T \approx 1905 \frac{(0.45+L)}{Q}+7.56
$$

It must be stated that the above equation has errors of 2.63s compared with Equations 4 and 5 , but as the period that derived from the experimental data is larger than 100 s which indicates that the error should be lower than $3 \%$, so it can be used to estimate and predict the period of oscillation curve in such circulating system. Although we have found the relationship between the period and the two experimental parameters, the length of the tube and the flow rate, the mechanism of it still needs to be found. As mentioned above, the attenuated oscillation is caused by the parabolic velocity profile in the experiment section. For single particle movement, due to the different height of particles in the tube, they should move at different velocity. However, from the viewpoint of particle group, the initial movement behavior of particle group can be approximately considered as the overall movement at a special velocity, and this velocity must have close link with the flowrate of suspension. In addition, for the properties of circulating flow, the overall movement of the particles leads to a repeated occurrence of particle group in the same position in the tube, which could finally lead to the oscillation of particle volume fraction. This is supposed to be the main reason why the period of the oscillation curve is linearly related to the length of the pipeline, but inversely related to the flow. After all, the particles are moving at different velocities, and their velocities are also changing with the position dynamically, which leads to the breakup of the overall migration of particles. Therefore, the particles could be re-distributed in different positions of the tube, which cause the attenuation of the oscillation 
curve.

As mentioned above, a time of $5 \mathrm{~T}$ is an important criterion for the particle suspension to reach the quasi-steady state. And we also believe that it is very valuable to the photocatalytic reaction, because the time of circulating flow is related to the working time of pump and a consumption of energy. In some practical photocatalytic reactor, the operation mode is intermittent and it only need to use pump when the suspension needs to be suspended again ${ }^{33}$. Therefore, we recommend that the pump only need to work for a time of $5 \mathrm{~T}$ and the particle suspension will be well suspended. It is worth noting that, according to Equation 6, the period T should be different in other cases having different tube diameter or length. Nevertheless, we suggest a simple method to evaluate $\mathrm{T}$ based on our experiment setup. According to Figure $4 \mathrm{a}$ and $\mathrm{d}$, the first peak and the second peak of the particle volume fraction in the circulating flow has an obvious correlation which could be distinguished clearly. It is therefore possible to measure the time between two peaks to evaluate the period T. And due to the wide application of such kind of nanoscale particle-laden flow, our method can thus be also used to predict the transport process in similar applications.

\section{Conclusions}

In this study, an experimental study is conducted to explain the transport phenomenon of nanoparticles in circulating flow. It can be found the particle volume fraction varies with time and appears periodical attenuated oscillation, which is closely related to the velocity distribution in laminar flow. And we further analyze the period of the attenuated oscillation curve by conducting a linear fitting, which shows that the period can be express as $T=f(Q, L)$ in which the period is proportional to the length of experiment section and inversely proportional to the flowrate. Also, we found the time $5 \mathrm{~T}$ is a very important criterion for whether the particle suspension has reached the quasi-steady state. And it is believed to be valuable to reduce energy consumption in the photocatalytic reaction.

In general, our obtained results are believed to be of value for the understanding of the nanoscale particle transport phenomenon in many natural or industrial processes. In particular, our results are helpful for the understanding and then effective control of the movement and 
distribution of photocatalyst particles in the tubular photocatalytic reactor, which is believed to significantly affect the energy conversion efficiency of the photocatalytic processes.

\section{Acknowledgements}

The authors gratefully acknowledge the financial support of the National Natural Science Foundation of China (No. 51776165, 51888103) and the financial support from Royal Society-Newton Advanced Fellowship grant (NAF\R1\191163).

\section{Literature cited}

1. Li, S.; Zhou, J.; Wang, Y.; Teng, A.; Zhang, K.; Wu, Z.; Cheng, S.; Wang, W., Physicochemical and Antimicrobial Properties of Hydroxypropyl Methylcellulose-Cinnamon Essential Oil Emulsion: Effects of Micro-and Nanodroplets. International Journal of Food Engineering 2019, 15, (9).

2. E, W.; Cheng, J.; Yang, C.; Mao, Z., Experimental study by online measurement of the precipitation of nickel hydroxide: Effects of operating conditions. Chinese Journal of Chemical Engineering 2015, 23, (5), 860-867.

3. Heath, A. R.; Fawell, P. D.; Bahri, P. A.; Swift, J. D., Estimating Average Particle Size by Focused Beam Reflectance Measurement (FBRM). Particle \& Particle Systems Characterization 2002, 19, (2), 84-95.

4. Jing, D. W.; Song, D. X., Optical properties of nanofluids considering particle size distribution: Experimental and theoretical investigations. Renewable and Sustainable Energy Reviews 2017, 78, 452-465.

5. Song, R.; Luo, B.; Liu, M. C.; Geng, J. F.; Jing, D. W.; Liu, H., Synergetic coupling of photo and thermal energy for efficient hydrogen production by formic acid reforming. AIChE Journal 2017, 63, (7), 2916-2925.

6. Jing, D. W.; Liu, H.; Zhang, X. H.; Zhao, L.; Guo, L. J., Photocatalytic hydrogen production under direct solar light in a CPC based solar reactor: Reactor design and preliminary results. Energy Conversion and Management 2009, 50, (12), 2919-2926.

7. Hisatomi, T.; Domen, K., Reaction systems for solar hydrogen production via water splitting with particulate semiconductor photocatalysts. Nature Catalysis 2019, 2, (5), 387-399.

8. Malato, S.; Blanco, J.; Campos, A.; Cáceres, J.; Guillard, C.; Herrmann, J. M.; Fernández-Alba, A. R., Effect of operating parameters on the testing of new industrial titania catalysts at solar pilot plant scale. Applied Catalysis B, Environmental 2003, 42, (4), 349-357.

9. Amiri, H.; Ayati, B.; Ganjidoust, H., Mass transfer phenomenon in photocatalytic cascade disc reactor: Effects of artificial roughness and flow rate. Chemical Engineering and Processing: Process Intensification 2017, 116, 48-59.

10. Banić, N. D.; Abramović, B. F.; Šojić, D. V.; Krstić, J. B.; Finčur, N. L.; Bočković, I. P., Efficiency of neonicotinoids photocatalytic degradation by using annular slurry reactor. Chemical Engineering Journal 2016, 286, 184-190.

11. Li Puma, G.; Yue, P. L., Enhanced photocatalysis in a pilot laminar falling film slurry reactor. Industrial \& Engineering Chemistry Research 1999, 38, (9), 3246-3254.

12. Barresi, A.; Baldi, G., Solid dispersion in an agitated vessel effect of particle shape and density. 
Chemical Engineering Science 1987, 12, (42), 2969-2972.

13. Huang, J. K.; Lu, Y. J.; Wang, H., A new quantitative measurement method for mixing and segregation of binary-mixture fluidized bed by capacitance probe. Chemical Engineering Journal 2017, 326, 99-108.

14. Su, X.; Wang, C.; Pei, H.; Li, J.; Lan, X.; Gao, J., Experimental Study of Solids Motion in an 18 m Gas-Solids Circulating Fluidized Bed with High Solids Flux. Industrial \& Engineering Chemistry Research 2019, 58, (51), 23468-23480.

15. Wang, K.; Liu, G.; Liu, Z. G.; Wu, J.; Yi, L. T.; Zhang, J. L.; Du, J. C., Acoustic sensor approaches for sand detection in sand - water two-phase flows. Powder Technology 2017, 320, 739-747.

16. Nocentini, M.; Pinelli, D.; Magelli, F., Dispersion coefficient and settling velocity of the solids in agitated slurry reactors stirred with multiple rushton turbines. Chemical Engineering Science 2002, 57 , (11), 1877-1884.

17. Jung, S. H.; Kim, J. S.; Kim, J. B.; Kwon, T. Y., Flow-rate measurements of a dual-phase pipe flow by cross-correlation technique of transmitted radiation signals. Applied Radiation and Isotopes 2009, 67, (7-8), 1254-8.

18. Gomes, F. P. C.; Garg, A.; Mhaskar, P.; Thompson, M. R., Data-Driven Advances in Manufacturing for Batch Polymer Processing Using Multivariate Nondestructive Monitoring. Industrial \& Engineering Chemistry Research 2019, 58, (23), 9940-9951.

19. Bieberle, A.; Schubert, M.; Da Silva, M. J.; Hampel, U., Measurement of Liquid Distributions in Particle Packings Using Wire-Mesh Sensor versus Transmission Tomographic Imaging. Industrial \& Engineering Chemistry Research 2010, 49, (19), 9445-9453.

20. Liu, L.; Fang, Z. Y.; Wu, Y. P.; Lai, X. P.; Wang, P.; Song, K., Experimental investigation of solid-liquid two-phase flow in cemented rock-tailings backfill using Electrical Resistance Tomography. Construction and Building Materials 2018, 175, 267-276.

21. Warsito, W.; Fan, L. S., ECT imaging of three-phase fluidized bed based on three-phase capacitance model. Chemical Engineering Science 2003, 58, (3-6), 823-832.

22. Black, D. L.; McQuay, M. Q.; Bonin, M. P., Laser-based techniques for particle-size measurement: A review of sizing methods and their industrial applications. Progress in Energy and Combustion Science 1996, 22, (3), 267-306.

23. Lourenço, A.; Arnold, J.; Cayre, O. J.; Rasteiro, M. G., Flocculation Treatment of an Industrial Effluent: Performance Assessment by Laser Diffraction Spectroscopy. Industrial \& Engineering Chemistry Research 2018, 57, (7), 2628-2637.

24. Ma, Z. H.; Merkus, H. G.; de Smet, J.; Heffels, C.; Scarlett, B., New developments in particle characterization by laser diffraction: size and shape. POWDER TECHNOLOGY 2000, 111, (1-2), 66-78.

25. Ochoa-Gutiérrez, K. S.; Tabares-Aguilar, E.; Mueses, M. Á.; Machuca-Martínez, F.; Li Puma, G., A Novel Prototype Offset Multi Tubular Photoreactor (OMTP) for solar photocatalytic degradation of water contaminants. Chemical Engineering Journal 2018, 341, 628-638.

26. Li, D.; Xiong, K.; Li, W.; Yang, Z. H.; Liu, C.; Feng, X.; Lu, X. H., Comparative Study in Liquid-Phase Heterogeneous Photocatalysis: Model for Photoreactor Scale-Up. Industrial \& Engineering Chemistry Research 2010, 49, (18), 8397-8405.

27. Malato, S.; Blanco, J.; Vidal, A.; Richter, C., Photocatalysis with solar energy at a pilot-plant scale: an overview. Applied Catalysis B-Environmental 2002, 37, (1), 1-15. 
28. Segré, G.; Silberberg, A., Behaviour of macroscopic rigid spheres in Poiseuille flow Part 1. Determination of local concentration by statistical analysis of particle passages through crossed light beams. Journal of Fluid Mechanics 1962, 14, (01), 115.

29. Tirumkudulu, M.; Tripathi, A.; Acrivos, A., Particle segregation in monodisperse sheared suspensions. Physics of Fluids 1999, 11, (3), 507-509.

30. Koh, C. J.; Hookham, P.; Leal, L. G., An experimental investigation of concentrated suspension flows in a rectangular channel. Journal of Fluid Mechanics 1994, 266, 1-32.

31. Hampton, R. E.; Mammoli, A. A.; Graham, A. L.; Tetlow, N.; Altobelli, S. A., Migration of particles undergoing pressure-driven flow in a circular conduit. Journal of Rheology 1997, 41, (3), 621-640.

32. Geng, J. F.; Wang, Y. C.; Hu, X. W.; Jing, D. W., Insights into the hydrodynamic properties of slurry flow in a tubular photocatalytic reactor by PIV combined with LSIA. Chemical Engineering Science 2016, 152, 127-139.

33. Cao, F.; Wei, Q. Y.; Liu, H.; Lu, N.; Zhao, L.; Guo, L. J., Development of the direct solar photocatalytic water splitting system for hydrogen production in Northwest China: Design and evaluation of photoreactor. Renewable Energy 2018, 121, 153-163.

34. Wei, Q. Y.; Yang, Y.; Hou, J. Y.; Liu, H.; Cao, F.; Zhao, L., Direct solar photocatalytic hydrogen generation with CPC photoreactors: System development. Solar Energy 2017, 153, 215-223.

35. Jing, D. W.; Guo, L. J.; Zhao, L.; Zhang, X. M.; Liu, H.; Li, M. T.; Shen, S. H.; Liu, G. J.; Hu, X. W.; Zhang, X. H., Efficient solar hydrogen production by photocatalytic water splitting: From fundamental study to pilot demonstration. International Journal of Hydrogen Energy 2010, 35, (13), 7087-7097.

36. Bello Lamo, M.; Buering, M.; Bahnemann, D., Effect of flowrate, photocatalyst loading and illumination conditions on the photocatalytic disinfection of recombinant Escherichia coli. Materials Research Innovations 2014, 19, (1), 20-23.

37. Budwig, R., Refractive index matching methods for liquid flow investigations. Experiments in Fluids 1994, 17, (5), 350-355.

38. Mukund, V.; Hof, B., The critical point of the transition to turbulence in pipe flow. Journal of Fluid Mechanics 2018, 839, 76-94.

39. Kalantary, R. R.; Shahamat, Y. D.; Farzadkia, M.; Esrafili, A.; Asgharnia, H., Photocatalytic degradation and mineralization of diazinon in aqueous solution using nano- $\mathrm{TiO}_{2}$ (Degussa, $\left.\mathrm{P} 25\right)$ : kinetic and statistical analysis. Desalination and Water Treatment 2015, 55, (2), 555-563.

40. Tolosana-Moranchel, A.; Casas, J. A.; Carbajo, J.; Faraldos, M.; Bahamonde, A., Influence of $\mathrm{TiO} 2$ optical parameters in a slurry photocatalytic reactor: Kinetic modelling. Applied Catalysis B: Environmental 2017, 200, 164-173.

41. Kowalczuk, P. B.; Drzymala, J., Physical meaning of the Sauter mean diameter of spherical particulate matter. Particulate Science and Technology 2016, 34, (6), 645-647.

42. Wang, L.; Fang, N. F.; Yue, Z. J.; Shi, Z. H.; Hua, L., Raindrop Size and Flow Depth Control Sediment Sorting in Shallow Flows on Steep Slopes. Water Resources Research 2018, 54, (12), 9978-9995.

43. Mahdi Jafari, S.; He, Y.; Bhandari, B., Nano-Emulsion Production by Sonication and Microfluidization-A Comparison. International Journal of Food Properties 2006, 9, (3), 475-485.

44. Melling, A., Tracer particles and seeding for particle image velocimetry. Measurement Science and Technology 1997, 8, (12), 1406-1416. 
45. Suarez, S.; Jansson, I.; Ohtani, B.; Sanchez, B., From titania nanoparticles to decahedral anatase particles: Photocatalytic activity of TiO2/zeolite hybrids for VOCs oxidation. Catalysis Today 2019, $326,(1), 2-7$.

46. Xingcai, L.; Kun, N., Effectively predict the solar radiation transmittance of dusty photovoltaic panels through Lambert-Beer law. Renewable Energy 2018, 123, 634-638.

47. Kowalkowski, T.; Buszewski, B.; Cantado, C.; Dondi, F., Field-Flow Fractionation: Theory, Techniques, Applications and the Challenges. Critical Reviews in Analytical Chemistry 2006, 36, (2), 129-135. 


\section{Table of Contents graphic}

An experimental set-up based on photocatalytic application was developed to investigate the nanoparticle volume fraction and size distribution in a laminar particle-laden circulating flow. A periodical attenuated oscillation of the particle volume fraction and mean particle size with time was found. It was also found by a fitting analysis that the period of the attenuated oscillation is related to the flowrate and length of experiment section, and the time of five periods is found to be an important criterion for particle control.

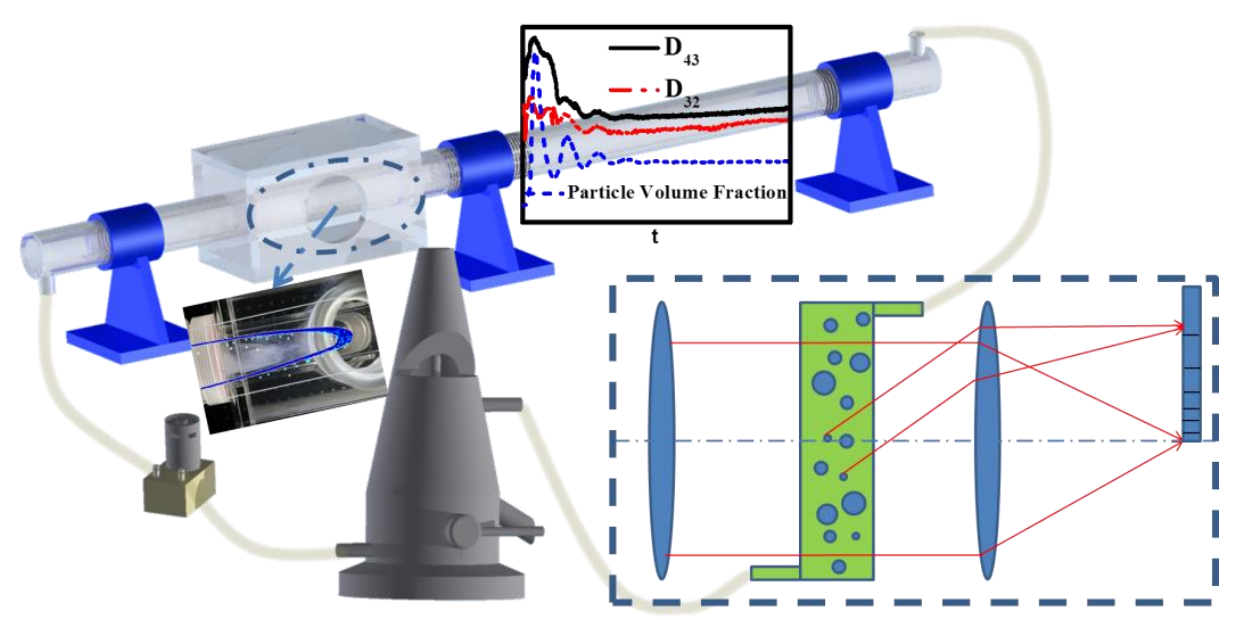

